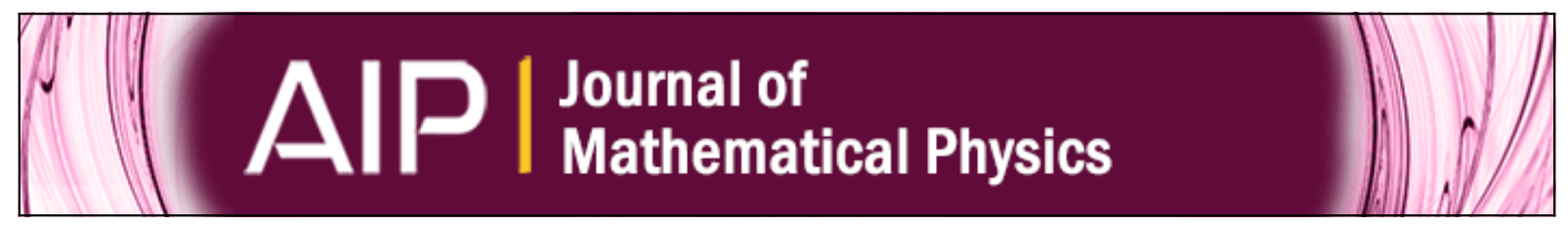

\title{
Non-homogeneous solutions of a Coulomb Schrödinger equation as basis set for scattering problems
}

J. A. Del Punta, M. J. Ambrosio, G. Gasaneo, S. A. Zaytsev, and L. U. Ancarani

Citation: Journal of Mathematical Physics 55, 052101 (2014); doi: 10.1063/1.4874115

View online: $\mathrm{http}: / / \mathrm{dx}$.doi.org/10.1063/1.4874115

View Table of Contents: http://scitation.aip.org/content/aip/journal/jmp/55/5?ver=pdfcov

Published by the AIP Publishing

\section{Articles you may be interested in}

Numerical Solution Of The TimeDependent Schrödinger equation

AIP Conf. Proc. 1150, 396 (2009); 10.1063/1.3192278

MeshFree Radial Basis Functions Method for the Accurate Numerical Solution of the Radial Schrödinger Equation: IBound States

AIP Conf. Proc. 1072, 241 (2008); 10.1063/1.3039838

Phase-amplitude method for numerically exact solution of the differential equations of the two-center Coulomb problem

J. Math. Phys. 43, 2169 (2002); 10.1063/1.1465098

Erratum: "Irreducible Brillouin conditions and contracted Schrödinger equations for n-electron systems. I. The equations satisfied by the density cumulants" [J. Chem. Phys. 114, 2047 (2001)]

J. Chem. Phys. 114, 8226 (2001); 10.1063/1.1362325

Irreducible Brillouin conditions and contracted Schrödinger equations for n-electron systems. I. The equations satisfied by the density cumulants

J. Chem. Phys. 114, 2047 (2001); 10.1063/1.1337058

\section{AlP Re-register for Table of Content Alerts}




\title{
Non-homogeneous solutions of a Coulomb Schrödinger equation as basis set for scattering problems
}

\author{
J. A. Del Punta, ${ }^{1}$ M. J. Ambrosio, ${ }^{1}$ G. Gasaneo, ${ }^{1}$ S. A. Zaytsev, ${ }^{2}$ \\ and L. U. Ancarani ${ }^{3}$ \\ ${ }^{1}$ Departamento de Física, Universidad Nacional del Sur, 8000 Bahía Blanca, \\ Buenos Aires, Argentina \\ ${ }^{2}$ Physics Department, Pacific National University, Khabarovsk 680035, Russia \\ ${ }^{3}$ Théorie, Modélisation, Simulation, SRSMC, UMR CNRS 7565, Université de Lorraine, \\ 57078 Metz, France
}

(Received 11 February 2014; accepted 17 April 2014; published online 20 May 2014)

We introduce and study two-body Quasi Sturmian functions which are proposed as basis functions for applications in three-body scattering problems. They are solutions of a two-body non-homogeneous Schrödinger equation. We present different analytic expressions, including asymptotic behaviors, for the pure Coulomb potential with a driven term involving either Slater-type or Laguerre-type orbitals. The efficiency of Quasi Sturmian functions as basis set is numerically illustrated through a two-body scattering problem. (c)2014 AIP Publishing LLC. [http://dx.doi.org/10.1063/1.4874115]

\section{INTRODUCTION}

Scattering problems are of fundamental importance in atomic physics. While two, three, or more particles can be involved, in many cases the whole collision process can be reduced to the study of two interacting particles. In other cases, and increasing the difficulty, the problem may be reduced to the dynamics of three interacting particles. Within quantum mechanics two-body scattering problems can be dealt with any degree of numerical accuracy, and can be considered as solved even when, in most of the cases, no analytic solution is known. When dealing with a scattering problem involving three particles something similar can be stated. This is mostly due to the great progress achieved in the last decade with ever increasing supercomputers capabilities. The simplest ionization process involving a three-body problem is electron impact ionization of hydrogen. In 1999, using exterior complex scaling, it was shown that all the information about this process can be extracted directly form the three-body wave function and remarkable agreement with experimental data can be found. ${ }^{1}$ The fact that the three-body scattering problem can be solved numerically is now well established, and the agreement found between the different approaches is remarkable (see the review ${ }^{2}$ ). Within methods like the convergent close coupling, ${ }^{2}$ the exterior complex scaling ${ }^{1,3}$ and the time dependent close coupling, ${ }^{4}$ among others, numerical recipes were developed leading to the exact solution of the problem when, e.g., the number of basis functions or of grid points is increased. When dealing with a larger number of particles, even in simple cases such as the electron impact double ionization of the helium atom, these methods still apply but limitations appear because of the huge computational resources required.

The study of a three-body, or $n$-body, scattering problem has two main ingredients. The wave function of the system must satisfy the Schrödinger equation, and asymptotically it must include a prepared initial state and final (generally) purely outgoing states. While this is well known from the fundamental point of view, it is not clear how to proceed in practice. Only few methods are able to numerically build the correct time-independent solution: most of them use two-body basis functions to construct the three-body correlation. Some of the methods, as the convergent close coupling 5,6 and the $J$-matrix, ${ }^{7}$ do so by imposing single continuum asymptotic conditions. Alternatively, the Generalized Sturmian Functions (GSF) method, ${ }^{8,9}$ enforce purely outgoing behavior on a square 
configuration space. According to the authors of Refs. 1, 3, and 10, explicit imposition of asymptotic conditions is completely avoided when using the exterior complex scaling approach. The present paper does not aim to address directly these issues, but rather to contribute in understanding how the solution of the three-body Schrödinger equation, and in particular its asymptotic behavior, is built up through the use of two-body functions. To do so, we introduce and study here novel two-body basis functions, named Quasi-Sturmian (QS), which have the following characteristics: (i) they are analytic, (ii) they diagonalize the Coulomb interaction, and (iii) they have Coulomb type outgoing asymptotic behavior. Such basis functions are alternative to the GSF, and constitute a tool that is expected to be useful to deal with both solving the three-body Schrödinger equation and imposing the asymptotic behavior on it.

To make clear the point about three-body issues, let us briefly present the type of equation that has to be solved together with the required asymptotic behavior. For the sake of presentation simplicity, let us consider the very well known $S$-wave three-body Temkin-Poet model for electron impact ionization of hydrogen (nuclear charge $Z=1$ ). ${ }^{11,12}$ This relatively simple model has been solved numerically and used to test different methodologies, e.g., the convergent-close coupling, ${ }^{13}$ exterior complex scaling, ${ }^{14}$ time dependent close coupling, ${ }^{15}$ among others. The standard three-body Schrödinger equation can be transformed into a driven one

$$
\left[-\frac{1}{2 r_{1}^{2}} \frac{\partial}{\partial r_{1}}\left(r_{1}^{2} \frac{\partial}{\partial r_{1}}\right)-\frac{1}{2 r_{2}^{2}} \frac{\partial}{\partial r_{2}}\left(r_{2}^{2} \frac{\partial}{\partial r_{2}}\right)-\frac{Z}{r_{1}}-\frac{Z}{r_{2}}+\frac{1}{r_{>}}-E_{a}\right] \phi_{\mathrm{sc}}^{+}\left(r_{1}, r_{2}\right)=\mathcal{F}\left(r_{1}, r_{2}\right),
$$

where $r_{1}, r_{2}$ are the electron coordinates, $r_{>}=\max \left[r_{1}, r_{2}\right]$, and $E_{a}$ is the system's energy. The source term $\mathcal{F}\left(r_{1}, r_{2}\right)$ is related to the chosen prepared initial state, and the asymptotic condition to be satisfied by the scattering wave function $\phi_{\mathrm{sc}}^{+}\left(r_{1}, r_{2}\right)$ is

$$
\phi_{\mathrm{sc}}^{+}\left(r_{1}, r_{2}\right) \rightarrow T_{k_{1}, k_{2}} \frac{e^{i\left[K \rho-\frac{C(\alpha)}{K} \ln (2 K \rho)\right]}}{\rho^{\frac{5}{2}}},
$$

where $\rho=\sqrt{r_{1}^{2}+r_{2}^{2}}, \tan \alpha=r_{2} / r_{1}, C(\alpha)$ is an angular dependent effective charge $\mathrm{e}^{5,9}$ and $K$ is the hyperspherical momentum. Equation (1) and the asymptotic condition (2) indicate clearly that the radial coordinates are coupled. From a practical (numerical) point of view, imposing the double continuum asymptotic behavior (2) to the scattering solution $\phi_{\mathrm{sc}}^{+}\left(r_{1}, r_{2}\right)$ is an important and difficult task (the situation is even more difficult for the full electron-hydrogen problem) that only few methods can perform. Two of them have explicitly shown the occurrence of such a behavior: the exterior complex scaling ${ }^{16}$ and the GSF method. With the latter, ${ }^{8,9,17,18}$ the solution is proposed as a linear combination of product of two-body GSFs

$$
\phi_{\mathrm{sc}}^{+}\left(r_{1}, r_{2}\right)=\sum_{n_{1}, n_{2}} a_{n_{1}, n_{2}}^{S} \frac{1}{r_{1} r_{2}} \frac{1}{\sqrt{2}}\left[S_{n_{1}}^{+}\left(r_{1}\right) S_{n_{2}}^{+}\left(r_{2}\right)+(-1)^{S} S_{n_{1}}^{+}\left(r_{2}\right) S_{n_{2}}^{+}\left(r_{1}\right)\right],
$$

where $a_{n_{1}, n_{2}}^{S}$ are linear coefficients for a given spin state $S$ (singlet or triplet). It is remarkable that, by imposing pure outgoing $(+)$ flux on each of the coordinates $r_{1}$ and $r_{2}$, the hyperspherical wave front of Eq. (2) is generated at large hyperradii $\rho$ as shown in Fig. 1 (a similar figure has been published, e.g., in Ref. 17, and also for a $S$-wave model of electron impact double ionization of helium ${ }^{19}$ ). For illustration purposes, we have chosen above the well-known Temkin-Poet electron-hydrogen ionization case, as it has been the most studied with several computational schemes. However, with expansion (3) modified appropriately to include angular coupling, ${ }^{9,18}$ the hyperspherical wave front builds up similarly for the real ionization process, and for other three-particle scattering problems including double ionization of helium by electron or photon impact. How this hyperspherical wave front, representing three-body correlation, is built up by the uncorrelated two-body basis set is not clear yet: this work is the first of a series conducted to understand this important asymptotic issue.

Two-body GSFs are constructed from interaction potentials which generally do not possess known analytic solutions. The basis sets are therefore generated numerically with now well established methods. ${ }^{18}$ The lack of analytical solutions makes it more difficult to understand why and how the hyperspherical wave front is generated by the two-body basis set, and generally to grasp the 


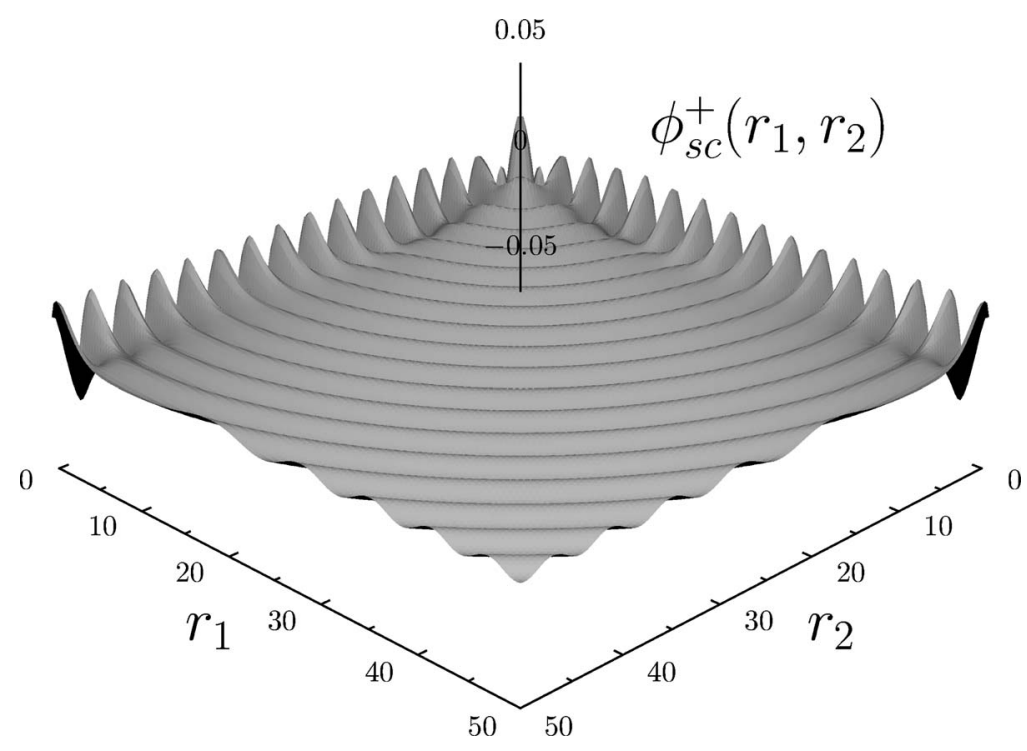

FIG. 1. Three-body scattering wave function for the Temkin-Poet electron hydrogen ionization, as a function of the two spherical electron-nucleus coordinates $r_{1}$ and $r_{2}$.

details of the three-body wave function. The present contribution offers and proposes, however, an alternative tool to work out the problem. We present different sets of basis functions - all analytical - having essentially the same properties as the GSFs: we named them QS. We are able to define such basis functions possessing any type of asymptotic condition (incoming, outgoing, and also standing wave) and, besides, being localized in a given configuration space region where the reaction is taking place. These properties are identical to those of the GSFs used, for example, to obtain the results shown in Fig. 1. In the present paper, the focus is placed on the derivation of different type of mathematical representations of the QS functions which should turn out to be useful for further three-body studies.

The QS basis functions introduced in this contribution can be obtained by directly solving nonhomogeneous equations either as done in Ref. 20 or by using the well known Green's methodology. When dealing with two-body problems, as we will discuss in this manuscript, the proposal may appear naive. However, the main objective behind it is the three-body problem. As explained above, the properties of the one coordinate basis functions induce the appropriate asymptotic behavior on the three-body case. Hence, this paper aims also to present all the necessary properties of the one-dimensional basis to be used, in a future contribution, for the description of the dynamics of more complicated problems, such as those appearing in single and double ionization of atoms by electron impact or absorption of radiation.

The paper is arranged as follows. In Sec. II we present the basic equations from which we derive the QS basis functions. Section III contains all the analytical formulas for the QS corresponding to a pure Coulomb potential; their asymptotic behavior is also provided in closed form. In Sec. IV, a numerical approach based on the finite difference method is also presented, and used to cross check the analytical formulas; the numerical method may be applied to any potential. In Sec. V, the efficiency of QS basis sets is illustrated with an application to a scattering problem for a Coulomb plus Yukawa potential. Finally, Sec. VI provides a summary and some perspectives.

Atomic units $(\hbar=e=1)$ are assumed throughout.

\section{STATEMENT OF THE PROBLEM AND PROPOSAL}

Let us consider the dynamic of a particle under the influence of a general potential $\bar{V}(r)$. For non-relativistic problems, and a given angular momentum $l$ and reduced mass $\mu$, this will be 
described by the solutions of the Schrödinger equation

$$
\left[-\frac{1}{2 \mu}\left(\frac{d^{2}}{d r^{2}}-\frac{l(l+1)}{r^{2}}\right)+\bar{V}(r)-E\right] \Psi^{+}(r)=0 .
$$

For scattering problems, the separation into initial $\Psi_{0}(r)$ plus scattering solution $\Psi_{\mathrm{sc}}^{+}(r)$ (outgoing behavior is assumed),

$$
\Psi^{+}(r)=\Psi_{0}(r)+\Psi_{\mathrm{sc}}^{+}(r),
$$

is generally proposed. This separation leads to the following driven equation for $\Psi_{\mathrm{sc}}^{+}(r)$

$$
\left[-\frac{1}{2 \mu}\left(\frac{d^{2}}{d r^{2}}-\frac{l(l+1)}{r^{2}}\right)+V(r)+U(r)-E\right] \Psi_{\mathrm{sc}}^{+}(r)=-U(r) \Psi_{0}(r),
$$

where we have separated the potential $\bar{V}(r)=V(r)+U(r)$ and assumed that $\Psi_{0}(r)$ is the solution of the Schrödinger equation with potential $V(r)$; the potential $U(r)$ represents the part of $\bar{V}(r)$ not solved by $\Psi_{0}(r)$ and should be in general of short range to be in agreement with the standard scattering theory.

In order to solve such scattering problems, we propose here a method that can be considered an extension of the standard Green's function method. Let us expand the right-hand side of (6) in a complete basis set $\varphi_{n, l}(r)$

$$
-U(r) \Psi_{0}(r)=\sum_{n} b_{n, l} \mathcal{U}_{\mathrm{g}}(r) \varphi_{n, l}(r),
$$

where the general function $\mathcal{U}_{\mathrm{g}}(r)$ is introduced with the purpose of convergence rate improvement. No requirement about orthogonality for $\varphi_{n, l}(r)$ is enforced and in general, for the studies to be performed here, it is not needed. Let us also expand the solution $\Psi_{\mathrm{sc}}^{+}(r)$ of $(6)$ as follows

$$
\Psi_{\mathrm{sc}}^{+}(r)=\sum_{n} a_{n, l} \psi_{n, l}^{+}(r)
$$

where the $\psi_{n, l}^{+}(r)$ functions satisfy the equation

$$
\left[-\frac{1}{2 \mu}\left(\frac{d^{2}}{d r^{2}}-\frac{l(l+1)}{r^{2}}\right)+V(r)-E\right] \psi_{n, l}^{+}(r)=\mathcal{U}_{\mathrm{g}}(r) \varphi_{n, l}(r) .
$$

This equation is similar to the generalized Sturmian equation ${ }^{9,18}$ (recall that Sturmian functions are eigensolutions of a Sturm-Liouville problem). However, instead of having the same function on the left and right hand sides, we have different functions. This is the reason for naming $\psi_{n, l}^{+}(r)$ Quasi-Sturmian functions.

Upon replacement of expansions (7) and (8) in the scattering equation (6), and using (9), we have

$$
\sum_{n} a_{n, l}\left[\mathcal{U}_{\mathrm{g}}(r) \varphi_{n, l}(r)+U(r) \psi_{n, l}^{+}(r)\right]=\sum_{n} b_{n, l} \mathcal{U}_{\mathrm{g}}(r) \varphi_{n, l}(r) .
$$

Projecting by the left with the functions $\varphi_{n^{\prime}, l}(r)$, we obtain a linear system for the coefficients $a_{n, l}$,

$$
(\mathbf{O}+\mathbf{U}) \mathbf{a}=\mathbf{O b},
$$

where $\mathbf{O}$ is the overlapping matrix between the basis functions $\varphi_{n, l}(r)$ with weight function $\mathcal{U}_{\mathrm{g}}(r)$,

$$
O_{n, n^{\prime}}=\int_{0}^{\infty} d r \varphi_{n^{\prime}, l}(r) \mathcal{U}_{\mathrm{g}}(r) \varphi_{n, l}(r),
$$

while the matrix elements of $\mathbf{U}$ are given by

$$
U_{n, n^{\prime}}=\int_{0}^{\infty} d r \varphi_{n^{\prime}, l}(r) U(r) \psi_{n, l}^{+}(r) .
$$

Solving the linear system (11) provides the coefficients $a_{n, l}$ and thus the scattering solution. We will show the efficiency of this proposal in Sec. V. 
One of the purposes of this contribution is to show that the QS functions corresponding to different $n$ values, and solutions of Eq. (9), form a complete basis set. We shall see that it is possible to generate basis functions possessing pure outgoing, incoming, or standing wave type behavior. Besides, for a Coulomb potential on the left-hand-side of (9), we will provide various properties and representations of the solutions corresponding to different choices of $\varphi_{n, l}(r)$ on the right-hand-side. The conclusions to be extracted from our investigation apply for any choice of $\varphi_{n, l}(r)$ defined in a finite region of the configuration space. However, to make the study explicit, we will assume that the function $\varphi_{n, l}(r)$ can be written in the quite general form

$$
\varphi_{n, l}(r)=e^{-\lambda r} r^{l+1} \sum_{j=0}^{M} a_{j, l}^{n} r^{j},
$$

where $M$ could be finite or infinite. Two important subcases to be discussed here are the Slater Type Orbitals (STO)

$$
\varphi_{n, l}^{\mathrm{STO}}(\lambda, r)=e^{-\lambda r} r^{l+n+1}
$$

and the Laguerre type (normalized) basis functions ${ }^{22}$

$$
\varphi_{n, l}^{L}(\lambda, r)=N_{n, l} e^{-\lambda r}(2 \lambda r)^{l+1} \frac{(2 l+2)_{n}}{n !}{ }_{1} F_{1}(-n, 2 l+2 ; 2 \lambda r),
$$

where

$$
N_{n, l}=\sqrt{\frac{n !}{\Gamma(2 l+2+n)}},
$$

and the Pochhammer symbol $(a)_{n}=\Gamma(a+n) / \Gamma(a)$ is defined in terms of the Gamma function. ${ }^{23}$ The basis functions (16) can be also viewed as a linear combination of STO

$$
\varphi_{n, l}^{L}(\lambda, r)=\sum_{s=0}^{n} c_{s, l}^{L} \varphi_{s, l}^{\mathrm{STO}}(\lambda, r)
$$

where

$$
c_{s, l}^{L}=N_{n, l} \frac{(2 \lambda)^{l+s+1}(2 l+2)_{n}(-n)_{s}}{n ! s !(2 l+2)_{s}} .
$$

The corresponding QS functions will be labelled with STO and $L$, respectively.

\section{ANALYTICAL QUASI STURMIAN FUNCTIONS FOR A PURE COULOMB POTENTIAL}

Let us consider the driven equation with a pure Coulomb potential

$$
\left[-\frac{1}{2 \mu}\left(\frac{d^{2}}{d r^{2}}-\frac{l(l+1)}{r^{2}}\right)+\frac{z_{1} z_{2}}{r}-E\right] \psi_{n, l}^{+}(r)=\mathcal{U}_{\mathrm{g}}(r) \varphi_{n, l}(\lambda, r) .
$$

Let the energy $E=k^{2} /(2 \mu)$ be positive, and the Sommerfeld parameter be defined by $\alpha=z_{1} z_{2} \mu / k$. Hereafter we define $\mathcal{U}_{\mathrm{g}}(r)=1 / r$ for convenience, but any positive (or null) power of $r$ can be equally treated.

\section{A. Multivariable hypergeometric representation of $\psi_{n, I}^{\mathrm{STO}+}(r)$ and $\psi_{n, I}^{L+}(r)$}

For the case in which we have $\varphi_{n, l}^{\mathrm{STO}}(\lambda, r)$ on the right-hand-side, the general solution of Eq. (20) with outgoing or incoming asymptotic behavior has been presented - in closed form - in Ref. 21. It is given as an appropriate sum of a particular solution of Eq. (20) and of the regular solution of the corresponding homogeneous differential equation (the regular Coulomb wave function ${ }^{22,24}$ )

$$
v_{l}^{\mathrm{Reg}}(r)=e^{-\pi \alpha / 2} \frac{|\Gamma(l+1+i \alpha)|}{2(2 l+1) !}(2 k r)^{l+1} e^{i k r}{ }_{1} F_{1}(l+1+i \alpha, 2 l+2 ;-2 i k r) .
$$


From Ref. 20, we know that a particular solution of this equation is given by

$$
\begin{aligned}
h_{n, l}(r)= & \frac{-2 \mu e^{i k r} r^{l+n+2}}{(n+1)(2 l+2+n)} \\
& \times \Theta^{(1)}\left(\begin{array}{c}
n+1,1 \mid 2 l+2+n, i \alpha+l+2+n \\
i \alpha+l+2+n \mid 2+n, 2 l+3+n
\end{array} \mid ;-(i k+\lambda),-2 i k r\right),
\end{aligned}
$$

where $\Theta^{(1)}$ is a two variables hypergeometric function introduced and discussed in Ref. 25 in a different context (the subscript of $\Theta^{(1)}$ has no useful meaning here, but we maintain the notation of Ref. 25), and whose series representation is given by

$$
\Theta^{(1)}\left(\begin{array}{c}
\mathfrak{a}_{1}, \mathfrak{a}_{2} \mid \mathfrak{b}_{1}, \mathfrak{b}_{2} \\
\mathfrak{c}_{1} \mid \mathfrak{d}_{1}, \mathfrak{d}_{2}
\end{array} \mid ; x_{1}, x_{2}\right)=\sum_{m_{1}=0}^{\infty} \sum_{m_{2}=0}^{\infty} \frac{\left(\mathfrak{a}_{1}\right)_{m_{1}}\left(\mathfrak{a}_{2}\right)_{m_{2}}\left(\mathfrak{b}_{1}\right)_{m_{1}}\left(\mathfrak{b}_{2}\right)_{m_{1}+m_{2}}}{\left(\mathfrak{c}_{1}\right)_{m_{1}}\left(\mathfrak{d}_{1}\right)_{m_{1}+m_{2}}\left(\mathfrak{d}_{2}\right)_{m_{1}+m_{2}}} \frac{x_{1}^{m_{1}} x_{2}^{m_{2}}}{m_{1} ! m_{2} !} .
$$

Following the recipe provided in Ref. 21, we may thus construct the solution $\psi_{n, l}^{\mathrm{STO} \pm}(r)$ having outgoing/incoming behavior.

Another, equivalent, representation of $\psi_{n, l}^{\mathrm{STO} \pm}(r)$ can be obtained in terms of the partial Coulomb Green's function $G_{l}^{( \pm)}\left(k ; r, r^{\prime}\right)$ which satisfies

$$
\left[-\frac{1}{2 \mu}\left(\frac{d^{2}}{d r^{2}}-\frac{l(l+1)}{r^{2}}\right)+\frac{z_{1} z_{2}}{r}-E\right] G_{l}^{( \pm)}\left(k ; r, r^{\prime}\right)=\delta\left(r-r^{\prime}\right),
$$

whereby

$$
\psi_{n, l}^{\mathrm{STO} \pm}(r)=\int_{0}^{\infty} d r^{\prime} G_{l}^{( \pm)}\left(k ; r, r^{\prime}\right) \frac{1}{r^{\prime}} \varphi_{n, l}^{\mathrm{STO}}\left(\lambda, r^{\prime}\right)
$$

The Green's function can be expressed as (see, e.g., Ref. 26)

$$
G_{l}^{( \pm)}\left(k ; r, r^{\prime}\right)=\mp \frac{\mu}{i k} \frac{\Gamma(l+1 \pm i \alpha)}{(2 l+1) !} \mathcal{M}_{\mp i \alpha ; l+1 / 2}\left(\mp 2 i k r_{<}\right) \mathcal{W}_{\mp i \alpha ; l+1 / 2}\left(\mp 2 i k r_{>}\right) .
$$

Then, using the integral representation (formula (6.669) of Ref. 23) for the product of the two Whittaker functions $\mathcal{M}$ and $\mathcal{W}$, one obtains

$$
\begin{aligned}
G_{l}^{( \pm)}\left(k ; r, r^{\prime}\right)= & 2 \mu \sqrt{r r^{\prime}} \int_{0}^{\infty} d y e^{ \pm i k\left(r+r^{\prime}\right) \cosh (y)}\left[\operatorname{coth}\left(\frac{y}{2}\right)\right]^{\mp 2 i \alpha} \\
& \times I_{2 l+1}\left(\mp 2 i k \sqrt{r r^{\prime}} \sinh (y)\right),
\end{aligned}
$$

where $I_{2 l+1}$ is a Bessel function. ${ }^{24}$ After some change of variables and intermediate steps one finds

$$
\begin{aligned}
\psi_{n, l}^{\mathrm{STO} \pm}(r)= & r^{l+1} e^{-\lambda r} \frac{2 \mu(2 l+2)_{n}}{(\lambda \mp i k)^{n+1}} \\
& \times \int_{0}^{1} d z z^{n}(1-z)^{l \pm i \alpha}\left(1-\omega^{ \pm 1} z\right)^{l \mp i \alpha} e^{z[\lambda \pm i k] r}{ }_{1} F_{1}(-n, 2 l+2 ; X),
\end{aligned}
$$

where

$$
\begin{gathered}
\omega \equiv e^{i \zeta}=\frac{\lambda+i k}{\lambda-i k} . \\
X=\frac{-r(1-z)\left(1-\omega^{ \pm 1} z\right)(\lambda \mp i k)}{z} .
\end{gathered}
$$

Expanding in series the confluent hypergeometric function (it is actually a polynomial of order $n$ ) appearing in (28), the integration over the variable $z$ can be performed using formula (3.385) of 
Ref. 23. Collecting we have

$$
\begin{aligned}
\psi_{n, l}^{\mathrm{STO} \pm}(r)= & r^{l+1} e^{-\lambda r} \frac{2 \mu(2 l+2)_{n} n !}{(\lambda \mp i k)^{n+1}(l+1 \pm i \alpha)_{n+1}} \sum_{p=0}^{n} \frac{(l+1 \pm i \alpha)_{p}}{(2 l+2)_{p}} \frac{(r(\lambda \mp i k))^{p}}{p !} \\
& \times \Phi_{1}\left(n-p+1,-(p+l \mp i \alpha), n+2+l \pm i \alpha ; \omega^{ \pm 1}, r(\lambda \pm i k)\right),
\end{aligned}
$$

where $\Phi_{1}\left(\mathfrak{a}_{1}, \mathfrak{b}_{1}, \mathfrak{c}_{1} ; x_{1}, x_{2}\right)$ is one of Horn's functions ${ }^{23}$ which can be expressed in several ways, for example, as an infinite sum of confluent hypergeometric functions (see below, Eq. (38)). We encounter - again - a two variable hypergeometric function, albeit relatively more familiar than the above $\Theta^{(1)}$.

If we now replace on the RHS of Eq. (20), the $\varphi_{n, l}^{\mathrm{STO}}(\lambda, r)$ by a Laguerre basis function $\varphi_{n, l}^{L}(\lambda, r)$, the sought QS solutions are directly provided by the linear combination (18)

$$
\psi_{n, l}^{L \pm}(r)=\sum_{s=0}^{n} c_{s, l}^{L} \psi_{s, l}^{\mathrm{STO} \pm}(r)
$$

In summary, we have provided analytical forms for QS functions solving the Coulomb differential equation (20) with either STO or Laguerre basis functions in the driven term.

\section{B. Laguerre representation of $\psi_{n, I}^{L+}(r)$}

Clearly, for $\mathcal{U}_{\mathrm{g}}(r)=1 / r$ and $\varphi_{n, l}=\varphi_{n, l}^{L}$, a solution $\psi_{n, l}^{L \pm}(r)$ to the non-homogeneous equation (20) can be expanded in the Laguerre basis functions (16):

$$
\psi_{n, l}^{L \pm}(r)=\sum_{m=0}^{\infty} \varphi_{m, l}^{L}(\lambda, r) G_{m, n}^{l( \pm)}(k ; \lambda)
$$

where

$$
G_{m, n}^{l( \pm)}(k ; \lambda)=\int_{0}^{\infty} \int_{0}^{\infty} d r d r^{\prime} \frac{1}{r} \varphi_{m, l}^{L}(\lambda, r) G_{l}^{( \pm)}\left(k ; r, r^{\prime}\right) \frac{1}{r^{\prime}} \varphi_{n, l}^{L}\left(\lambda, r^{\prime}\right)
$$

are the matrix elements of the Green function operator (26). They have been evaluated by Heller ${ }^{27}$ (see also Ref. 28 and the references therein) in terms of the two independent J-matrix solutions ${ }^{29}$

$$
G_{n, n^{\prime}}^{l( \pm)}(k ; \lambda)=\frac{2 \mu}{k} \mathcal{S}_{n_{<}, l}(k) \mathcal{C}_{n_{>}, l}^{( \pm)}(k),
$$

where $n_{<}$and $n_{>}$are the lesser and greater of $n$ and $n^{\prime}$. Here the regular $\mathcal{S}_{n, l}$ and irregular $\mathcal{C}_{n, l}^{( \pm)}$ solutions are expressed in terms of the Pollaczek polynomials and Pollaczek functions of the second kind, respectively, as Refs. 29-31:

$$
\begin{aligned}
\mathcal{S}_{n, l}(k)= & e^{-\pi \alpha / 2} \frac{|\Gamma(l+1+i \alpha)|}{2 N_{n, l}(2 l+1) !}(2 \sin \zeta)^{l+1} \omega^{-i \alpha} \\
& \times(-\omega)^{n}{ }_{2} F_{1}\left(-n, l+1+i \alpha, 2 l+2 ; 1-\omega^{-2}\right), \\
\mathcal{C}_{n, l}^{( \pm)}(k)= & -\frac{n !}{N_{n, l}} \frac{e^{\pi \alpha / 2} \omega^{i \alpha}}{(2 \sin \zeta)^{l}} \frac{\Gamma(l+1 \pm i \alpha)}{|\Gamma(l+1 \pm i \alpha)|} \\
& \times \frac{(-\omega)^{ \pm(n+1)}}{\Gamma(n+l+2 \pm i \alpha)}{ }_{2} F_{1}\left(-l \pm i \alpha, n+1, n+l+2 \pm i \alpha ; \omega^{ \pm 2}\right),
\end{aligned}
$$

where $\zeta$ is defined through relation (29). For the Laguerre-type basis on the RHS, expansion (33), together with the analytical coefficients (35), provides an alternative formulation to that given by (32) constructed in terms of hypergeometric functions. 
In the standard J-matrix method (as presented in, e.g., Ref. 30), only the lowest $\varphi_{0, l}^{L}(r)$ is considered in the driven term. By allowing, as we do here, any Laguerre basis element on the RHS of the non-homogeneous equation, we may say that we somehow extend and generalize the methodology (see, e.g., Ref. 29).

\section{Asymptotic behavior of the basis functions}

Let us now focus on the asymptotic behavior of the QS function (31). For our purposes, a convenient series representation of the Horn $\Phi_{1}$ is the following: ${ }^{23}$

$$
\Phi_{1}(a, b, c ; x, y)=\sum_{q=0}^{\infty} \frac{(a)_{q}(b)_{q}}{(c)_{q}} \frac{x^{q}}{q !}{ }_{1} F_{1}(a+q, c+q ; y) .
$$

Then, for the STO case, the QS functions $\psi_{n, l}^{\mathrm{STO} \pm}(r)$ become

$$
\begin{aligned}
\psi_{n, l}^{\mathrm{STO} \pm}(r)= & r^{l+1} e^{-\lambda r} \frac{2 \mu(2 l+2)_{n} n !}{(\lambda \mp i k)^{n+1}(l+1 \pm i \alpha)_{n+1}} \sum_{p=0}^{n} \frac{(l+1 \pm i \alpha)_{p}}{(2 l+2)_{p}} \frac{(r(\lambda \mp i k))^{p}}{p !} \\
& \times \sum_{q=0}^{\infty} \frac{(n-p+1)_{q}(-(p+l \mp i \alpha))_{q}}{(n+2+l \pm i \alpha)_{q}} \frac{\omega^{ \pm q}}{q !} \\
& \times{ }_{1} F_{1}(n-p+1+q, n+2+l \pm i \alpha+q ; r(\lambda \pm i k)) .
\end{aligned}
$$

Taking the $r \rightarrow \infty$ limit of the ${ }_{1} F_{1}$ function, ${ }^{24}$ retaining only the exponential term, the two summations can be calculated in closed form. After some algebraic simplifications we obtain the following asymptotic behavior

$$
\psi_{n, l}^{\mathrm{STO} \pm}(r) \sim A_{l, n} e^{ \pm i\left(k r-\alpha \ln (2 k r)+\sigma_{l}-\frac{l \pi}{2}\right)},
$$

where the coefficient is seen to be independent of the \pm choice

$$
\begin{aligned}
A_{l, n}= & (\omega)^{-i \alpha} e^{-\frac{\pi}{2} \alpha} \frac{2 \mu(2 l+2)_{n}}{2 k(\lambda-i k)^{n}}|\Gamma(l+1 \pm i \alpha)|\left(\frac{2 k}{\lambda^{2}+k^{2}}\right)^{l+1} \\
& \times{ }_{2} F_{1}\left(-n, l+1+i \alpha, 2 l+2 ; 1-\omega^{-1}\right),
\end{aligned}
$$

and $\sigma_{l}=\operatorname{Arg}[\Gamma(l+1+i \alpha)]$ is the Coulomb phase.

For the Laguerre case, we need to consider a further summation (see Eq. (32)) which can also be calculated exactly. Algebraic steps lead to the following asymptotic behavior

$$
\psi_{n, l}^{L \pm}(r) \sim B_{n, l} e^{ \pm i\left(k r-\alpha \ln (2 k r)+\sigma_{l}-\frac{l \pi}{2}\right)},
$$

with the \pm independent coefficients

$$
\begin{aligned}
B_{n, l}= & N_{n, l} \frac{2 \mu}{k} e^{-\frac{\pi}{2} \alpha} \omega^{-i \alpha}\left(\frac{4 k \lambda}{\lambda^{2}+k^{2}}\right)^{l+1} \frac{(2 l+2)_{n}}{2 n !}|\Gamma(l+1 \pm i \alpha)|(-\omega)^{n} \\
& \times{ }_{2} F_{1}\left(-n, l+1+i \alpha, 2 l+2 ; 1-\omega^{-2}\right) .
\end{aligned}
$$

By comparison, one finds the connection $B_{n, l}=(2 \mu / k) \mathcal{S}_{n, l}(k)$ between these coefficients and those obtained from Green's method.

As shown through formulae (40) and (42), both the STO and Laguerre QS possess the correct Coulombic asymptotic behavior.

\section{General behavior of $\psi_{n, I}^{\mathrm{STO}}(r)$ and $\psi_{n, I}^{\mathrm{L \pm}}(r)$ as basis functions}

Let us illustrate the above analytical formulation of QS functions. Only outgoing behavior will be considered. We consider the case of a Coulomb potential with $z_{1} z_{2}=-1$ on the LHS of Eq. (20) and $\mathcal{U}_{\mathrm{g}}(r)=1 / r$ on the RHS. 

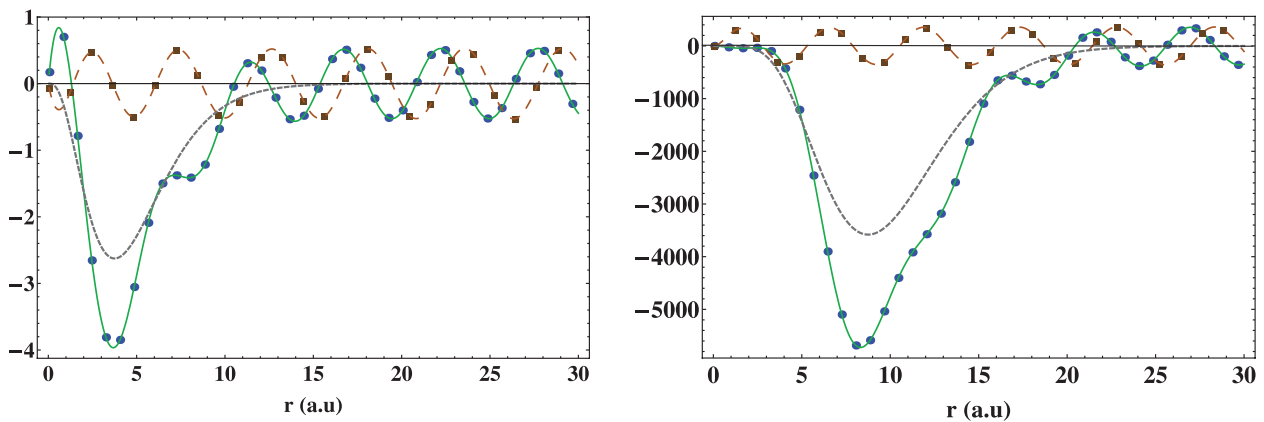

FIG. 2. Real and imaginary parts of QS functions corresponding to a Slater-type driven term with $\lambda=0.8, k=1.1$ (the RHS of Eq. (20) is shown with a dashed line). Left panel $(l=0, n=3)$ and right panel $(l=1, n=6)$. The analytical calculations are shown with solid circles (real part) and solid squares (imaginary part) and the numerical (finite difference scheme) with solid lines (real part) and long dashed lines (imaginary part).

We start with a Slater-type driven term. In Fig. 2, we have plotted as a function of $r$, the real and imaginary parts of the analytical QS for two sets: left panel $(l=0, n=3)$ and right panel $(l=1$, $n=6$ ). The analytical calculations coincide with the numerical ones obtained with a finite difference scheme (see Sec. IV). We clearly observe that, as soon as the driven term (the RHS of Eq. (20)) vanishes, the QS gain their pure outgoing behavior.

Next we consider a Laguerre type driven term. In Fig. 3, we have plotted as a function of $r$, the real and imaginary parts of the analytical QS for two sets: left panel $(l=1, n=7)$ and right panel $(l=2, n=4)$. The corresponding driven terms present several nodes; as soon as it vanishes the pure asymptotic behavior is taken by the QS functions. Again, the purely numerical and analytical calculations coincide.

We have evaluated Laguerre-type QS basis functions $\psi_{n, l}^{L+}(r)$ for different values of $n$; a sample is shown in Fig. 4 as a function of $r$. The different QS functions are clearly independent in the region where the driven term is still present, and can thus serve to build a basis set. To best appreciate the asymptotic behavior given by Eq. (42), the quantity $\psi_{n, l}^{L \pm}(r) / B_{n, l}$ rather than the QS functions $\psi_{n, l}^{L \pm}(r)$ are shown. When the driven term has vanished, all four solutions tend towards the same pure outgoing wave. Note that as $n$ increases this convergence is reached at larger values of $r$ (the same is observed with $\mathrm{GSF}^{9,18}$ ); this is further illustrated on the right panel where only the slowest cases $(n=19$ and $n=28)$ are shown.

A curious and interesting phenomenon was observed when the RHS of Eq. (20) matches, by coincidence, an eigenstate of the LHS potential. This may occur, for example, with a STO $\varphi_{1,0}^{\mathrm{STO}}(1, r)$ and $\mathcal{U}_{\mathrm{g}}(r)=1 / r$ providing a driven term which coincides with the hydrogen ground state of potential
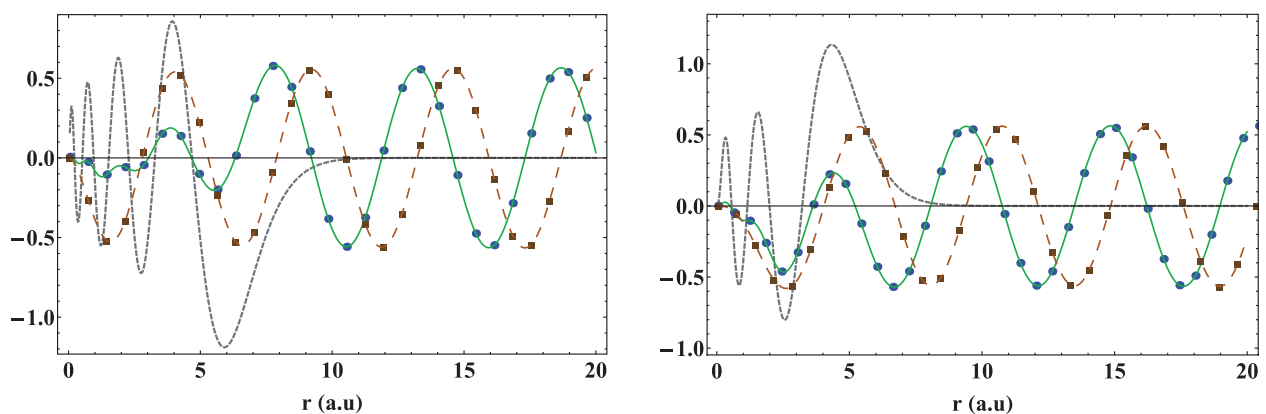

FIG. 3. Real and imaginary parts of QS functions corresponding to a Laguerre-type driven term with $\lambda=2.6, k=1.1$ (the RHS of Eq. (20) is shown with a dashed line). Left panel $(l=1, n=7)$ and right panel $(l=2, n=4)$. The analytical calculations are shown with solid circles (real part) and solid squares (imaginary part) and the numerical (finite difference scheme) with solid lines (real part) and long dashed lines (imaginary part). 

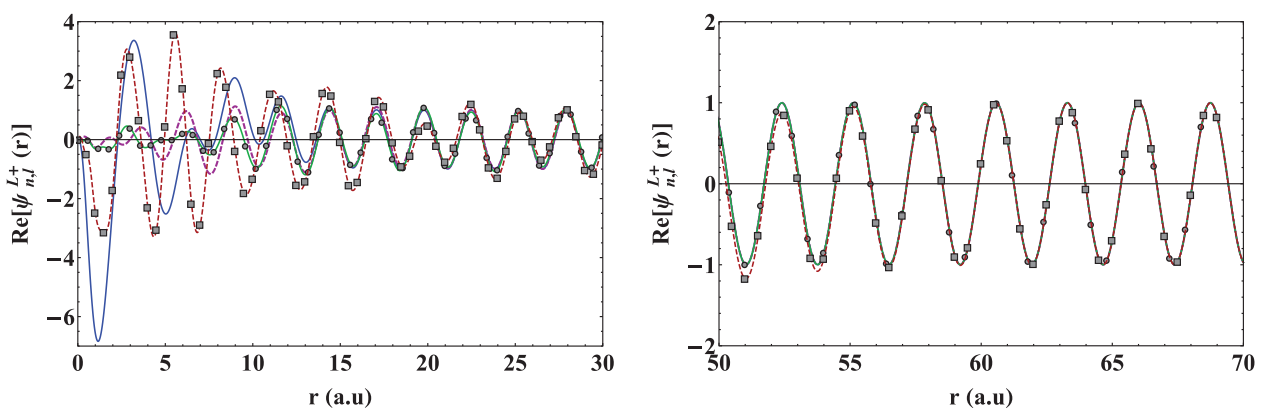

FIG. 4. Real part of four QS functions $(n=4,9,19,28)$ corresponding to a Laguerre-type driven term with $\lambda=1.2$, $k=2.3, l=2$. What is shown is $\psi_{n, l}^{L \pm}(r) / B_{n, l}$ for $n=4$ (solid line), $n=9$ (dotted line), $n=19$ (solid line with circles), and $n=28$ (dotted line with squares). Left panel: inner region. Right panel: asymptotic regime, where only the $n=19$ and $n=28$ cases are shown.

$V(r)=-1 / r$. As we now show, the resulting QS function copies - in a resonant manner - the shape of the RHS with a scaling factor which can be properly characterized.

To describe this, let us start from Eq. (9), and express the scattering solution and its RHS as linear combinations of the energy eigenstates $\xi_{\bar{E}}(r)$ of the potential $V(r)$

$$
\begin{gathered}
\psi_{n, l}^{+}(r)=\int d \bar{E} a_{\bar{E}} \xi_{\bar{E}}(r), \\
\mathcal{U}_{\mathrm{g}}(r) \varphi_{n, l}(\lambda, r)=\int d \bar{E} b_{\bar{E}} \xi_{\bar{E}}(r),
\end{gathered}
$$

where the integration encompasses both discrete and continuum states. Replacing such expansions in (9), a relation can be established between $a_{\bar{E}}$ and $b_{\bar{E}}$,

$$
\int a_{\bar{E}}[\bar{E}-E] \xi_{\bar{E}}(r) d \bar{E}=\int b_{\bar{E}} \xi_{\bar{E}}(r) d \bar{E}
$$

which implies

$$
a_{\bar{E}}=\frac{b_{\bar{E}}}{\bar{E}-E} .
$$

Now let us consider the unlikely but possible event where the RHS is proportional to an eigenstate of $V(r)$ with energy $E_{i}$. In this situation we would have $b_{\bar{E}}=\alpha \delta\left(\bar{E}-E_{i}\right)$. Then $a_{\bar{E}}=\frac{\alpha \delta\left(\bar{E}-E_{i}\right)}{\bar{E}-E}$, and the resulting QS, $\psi_{n, l}^{+}(r)$, is going to mimic the eigenstate $\xi_{E_{i}}(r)$, albeit scaled by the factor $\frac{\alpha}{E_{i}-E}$.

This resonant phenomenon is illustrated through the example shown in Fig. 5. The real part of QS, $\psi_{1,0}^{+}(r)$, are shown as a function of $r$, with $\varphi_{1,0}^{\mathrm{STO}}(\lambda, r)$ in the driven term, and for several $\lambda$ values close to 1 . Since we have taken $V(r)=-1 / r, \mathcal{U}_{\mathrm{g}}(r)=1 / r$ and $E=1.205$ a.u., when $\lambda=1$ the calculated QS is a scaled down copy of $\varphi_{1,0}^{\mathrm{STO}}(1, r)$ with a factor $\frac{1}{E_{i}-E}=-\frac{1}{1.705}$. As we step out of the resonant value, even by small amounts, the oscillations increase in amplitude very rapidly. Resonant elements may turn up as part of the basis; although they override the asymptotic behavior, they still appreciably shape the solution near the origin and should not therefore be discarded (this aspect will be more deeply discussed in a follow-up contribution). The resonance phenomenon provides a further application: it can be used to check whether either analytical evaluations or numerical implementations, such as the one covered in Sec. IV, are working properly.

\section{NUMERICAL QUASI STURMIAN FUNCTIONS}

Although in this manuscript our main objective is to provide analytic expressions for the QS functions (Sec. III), we also outline, within a finite difference approach, a general methodology to generate them numerically. On one hand, this will allow to cross-check the analytical results of Sec. III. On the other, the proposed numerical approach goes further as it is able to deal with an 


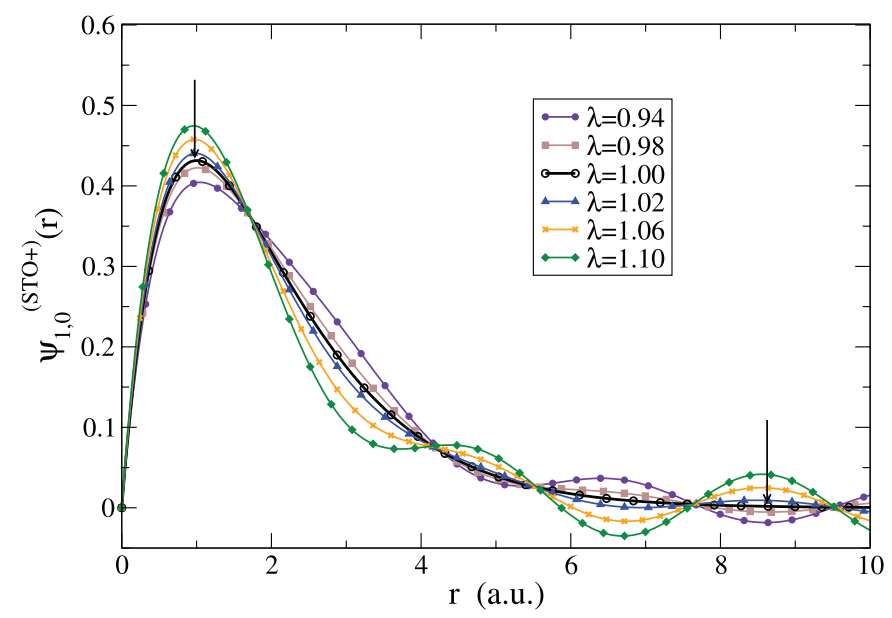

FIG. 5. Real part of QS functions $\psi_{1,0}^{\mathrm{STO}+}(r)$ with $\varphi_{1,0}^{\mathrm{STO}}(\lambda, r)$ and $\mathcal{U}_{\mathrm{g}}(r)=1 / r$ on the RHS, $V(r)=-1 / r$, and energy set to $E=1.205$ a.u. Different $\lambda$ values close to 1 are considered (lines with symbols - see inset). The $\lambda=1$ case (black, solid line with open circles) is a scaled down copy of the $\varphi_{1,0}^{\mathrm{STO}}(1, r)$ with a factor $-1 / 1.705$. Arrows are used to emphasize how the oscillation amplitude decreases as $\lambda \rightarrow 1$.

Eq. (9), with any kind of potential on the LHS and any basis functions $\varphi_{n, l}(\lambda, r)$ in the driven term. Such numerically obtained QS functions may then be used in sophisticated three-body problem calculations in a similar fashion as the generalized Sturmian functions have been used in, e.g., Refs. 8 and 17.

We thus propose to numerically solve the equation (here written for outgoing behavior)

$$
\left[-\frac{1}{2 \mu}\left(\frac{d^{2}}{d r^{2}}-\frac{l(l+1)}{r^{2}}\right)+\bar{V}(r)-E\right] \psi_{n, l}^{+}(r)=\mathcal{U}_{\mathrm{g}}(r) \varphi_{n, l}(\lambda, r)
$$

where we assume that $\bar{V}(r)$ is any kind of potential in an inner region and may have a Coulomb tail at large distances. We note that Eq. (9) is just a particular case of (48) where $\bar{V}(r)$ is simply $V(r)$.

We start by making a discretization of the radial coordinate as $r_{i}=i \Delta$, with $\Delta=r_{\max } / N$ and we consider a uniform grid with $i=0,1, \cdots, N+1$. Boundary conditions are enforced from the known behavior of the solutions at $r_{0}$ and $r_{N+1}$. The second derivative of the yet unknown function is approximated using Lagrangian differentiation with an odd $n_{s}$ number of points. Given any $r_{i}$ grid point between $r_{0}$ and $r_{N+1}$, we can pick a subset of $n_{s}$ points which contains that point. Then we can establish a relation for the second derivative at $r_{i}$ in terms of the values of the function at each of the subset points. Whenever possible we pick $\frac{n_{s}-1}{2}$ points on both sides of $r_{i}$ in order to get a centered differentiation expression. This is preferred because a Lagrangian approximant with uniform grid has the least error at the middle point of its domain. However, in the first and last $\frac{n_{s}-1}{2}$ points of the radial domain we cannot use a centered differentiation scheme trivially, because we would be requiring points beyond $r_{N+1}$ or unphysical $r<0$ ones. Let us address the issue near the origin first. We do know that the regularity condition imposes $\psi_{l, n}^{+}(0)=0$. Thus, for the first $i=1, \ldots, \frac{n_{s}-1}{2}$ points we are going to choose differentiation formulae involving the subset of $p=0, \ldots, n_{s}-1$. We now turn to the rightmost $\frac{n_{s}-1}{2}$ points. Since the asymptotic behavior of the QS function we want to generate is well established, we can still work with centered differentiation. We assume for the QS the outgoing behavior given by $H_{l}^{+}(r)$, the Coulomb wave function with pure outgoing behavior (note that the numerical method is general enough to impose any desired asymptotic behavior on the QS set).

All the resulting equations are recast as an $N \times N$ linear system, and solved with the routine ZGESV (which solves a linear system of equations with a square, complex matrix). ${ }^{32}$ This routine even enables to solve the equation for many different driven terms, just performing the matrix factorization only once. All numerical QS generated in this work were obtained with a finite difference scheme with $n_{s}=23$. This number provides a high accuracy, while still having the 
Lagrangian differentiation matrix construction unaffected by the finite machine precision. At around $n_{s}=31$ the finite algebra begins to hinder the quality of the differentiation matrix obtained by our procedure.

For the simplest finite difference scheme, namely, the case $n_{s}=3$, we use the routine ZGTSV (which is similar to the routine ZGESV but tailored for the specific case of a tridiagonal matrix). ${ }^{32}$ Although the finite difference scheme is of a lesser order, one can make up for that with a much denser grid and achieve similar precisions. The $n_{s}=3$ case leads to a tridiagonal matrix, and thus to a connection with the theory of orthogonal polynomials, since they also satisfy a tridiagonal recurrence relation. In a separate contribution we will delve deeper into the numerical schemes for generating QS functions, comparing their convergence rates as the radial coordinate is discretized into thinner intervals.

\section{APPLICATION TO A SCATTERING PROBLEM}

To illustrate the efficiency of the proposed QS basis in scattering problems, we solve here the scattering of a particle in a combined attractive Coulomb potential $V(r)=-2 / r\left(z_{1} z_{2}=-2\right)$ plus a Yukawa potential

$$
U(r)=-b \frac{e^{-a r}}{r} .
$$

We propose as a "free" solution $\Psi_{0}(r)$ the regular Coulomb solution $v_{l}^{\text {Reg }}(r)$ given by formula (21). This means that the driven term of Eq. (6) is given by the product of the potential (49) and the free solution (21). To solve this problem, various approaches were used and compared.

First, we used the finite difference technique of Sec. IV. In this case we generate two kinds of QS functions having, either the potential $\bar{V}(r)=z_{1} z_{2} / r+U(r)$ or only $\bar{V}(r)=z_{1} z_{2} / r$. In both cases we solved the QS equation including $\varphi_{m, l}^{L}(r)$ on the RHS.

Second, and for convergence rate comparison purposes only, we have used also the Generalized Sturmian method. ${ }^{33}$

Finally, we employed the analytical QS functions provided in Sec. III A and the Laguerre representation of $\psi_{n, l}^{L+}(r)$ described in Sec. III B. A linear system of equations as the one given by Eq. (11) was solved with the first technique. When the QS functions solve the whole potential, only a linear combination of QS functions with the coefficient corresponding to the $U(r) \Psi_{0}(r)$ is required. When the QS functions only include the Coulomb potential, the general methodology of Sec. II is required and used.

When using the Laguerre basis representation (i.e., expansion (8) with $\left.\psi_{n, l}^{L+}(r)\right)$ the linear system of equations (11) results to be

$$
\sum_{n=0}^{N}\left[\delta_{m n}+[U G]_{m, n}\right] a_{n}=b_{m} .
$$

Here the RHS array can be calculated explicitly and is given by

$$
\begin{aligned}
b_{m} \equiv & -\int_{0}^{\infty} d r \varphi_{m, l}^{L}(r) U(r) v_{l}^{\mathrm{Reg}}(r) \\
= & b\left[\frac{4 k \lambda}{(a+\lambda)^{2}+k^{2}}\right]^{l+1} e^{-\pi \alpha / 2} \frac{|\Gamma(l+1+i \alpha)|}{2 N_{m, l}(2 l+1) !}\left(\frac{a+\lambda-i k}{a+\lambda+i k}\right)^{i \alpha}\left(\frac{a-\lambda-i k}{a+\lambda-i k}\right)^{m} \\
& \times{ }_{2} F_{1}\left(-m, l+1+i \alpha, 2 l+2 ; \frac{-i 4 k \lambda}{a^{2}-(\lambda+i k)^{2}}\right) .
\end{aligned}
$$

The matrix elements $[U G]_{m, n}$ are defined by

$$
[U G]_{m, n}=\int_{0}^{\infty} d r \varphi_{m, l}(r) U(r) \psi_{n, l}^{L+}(r),
$$




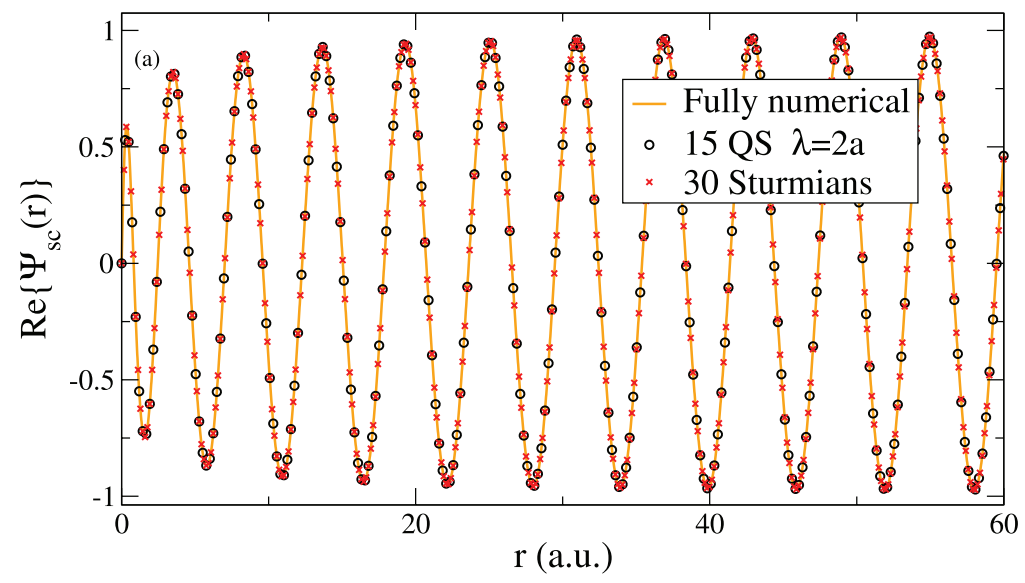

FIG. 6. Real part of the scattering solution for a Coulomb plus Yukawa type potential $\left(-2 / r-10 e^{-1.3 r} / r\right)$ obtained with 30 GSF (crosses), 15 QS, and with a finite difference scheme (solid line).

or, using (33) as proposed in Sec. III B,

$$
[U G]_{m, n}=\sum_{q=0}^{\infty} G_{q, n}^{l( \pm)}(k ; \lambda)\left\langle\varphi_{m, l}^{L}|U(r)| \varphi_{q, l}^{L}\right\rangle,
$$

the Green's function elements $G_{q, n}^{l( \pm)}(k ; \lambda)$ being given by (35). The matrix elements involving $U(r)$ are given analytically by

$$
\begin{aligned}
\left\langle\varphi_{m, l}^{L}|U(r)| \varphi_{n, l}^{L}\right\rangle= & -b \frac{1}{N_{n, l} N_{m, l}(2 l+1) !}\left(\frac{2 \lambda}{a+2 \lambda}\right)^{2 l+2}\left(\frac{a}{a+2 \lambda}\right)^{m+n} \\
& \times{ }_{2} F_{1}\left(-m,-n, 2 l+2 ;\left(\frac{2 \lambda}{a}\right)^{2}\right) .
\end{aligned}
$$

Numerically we truncate the summation (54) at $q=300$.

We performed explicit calculations with the following parameters $b=10, a=1.3, \lambda=2 a, z_{1} z_{2}$ $=-2, \mu=1, k=1, l=0$. In Fig. 6 we have plotted, as a function of $r$, the real part of the scattering solution $\Psi_{\mathrm{sc}}(r)$ obtained with $15 \mathrm{QS}, 30 \mathrm{GSF}$, and with our finite difference scheme described in Sec. IV.

Only 5 Laguerre basis functions are necessary to acceptably expand the driven term $U(r) \Psi_{0}(r)$, but more QS functions are necessary to appropriately represent the interaction $U(r)$ on the LHS of Eq. (6). To reach the same level of convergence, 15 QS were necessary whereas 30 GSF were needed. Both expansions are indistinguishable form each other, and from the finite difference result. QS asymptotic conditions can be tailored to suit those of the problem in question, just as generalized Sturmian sets. Neither set needs to strain themselves to expand the asymptotic regime, since they both have it already built in. This ultimately leads to a good converged solution without the need of large numbers of basis elements, the QS being even superior to the already very efficient GSF. One final statement: we have mentioned that the $\varphi_{n, l}(\lambda, r)$ set does not need to be orthogonal. As convergence is concerned, this factor alone does not define whether a $\varphi_{n, l}(\lambda, r)$ set is a better or worse choice. More important is the number of elements needed to expand the driven term of Eq. (6), and the fact that the set does not become numerically linearly dependent.

\section{SUMMARY AND PERSPECTIVES}

The results presented in this contribution can be separated in two types: physical and mathematical. 
In view of physical applications, we presented and discussed a generalization of the Green's method to solve scattering problems. We use the solutions (the QS) of a simpler problem, i.e., those of a non-homogeneous problem containing different types of basis functions on the RHS. As these elements can be used to expand any general function, the solution of the scattering problem may be constructed as a combination of the proposed QS functions. We showed that the latter are known analytically, and can be set to have any desired asymptotic behavior. The present investigation concentrated on the case corresponding to the Coulomb potential which is of fundamental importance for the implications it has on the much more complicated three-body Coulomb problem, the one we are ultimately interested in. In addition, we also presented a generalization of the J-matrix solutions. Indeed, only the case where the basis in the RHS correspond to $n=0$ is presented in the literature; here, we have given the solution for all $n$.

The mathematical results of the paper are associated to the extension of some results of a previous publication. ${ }^{20}$ In particular, we provided different representations based on two-variables hypergeometric functions for the solutions of the non-homogeneous Coulomb Schrödinger equation. In addition to such representations, we studied their asymptotic behaviors and gave expressions for the coefficients resulting for both STO and Laguerre basis on the RHS. Most of the mathematical analysis and the formulae provided were detailed for the case of the Coulomb potential. However, the basis characteristics can be considered, in general terms, valid also for other potentials. We showed that basis functions extending the standard Green's method can be defined. With that purposes in mind, we presented briefly a numerical procedure to be used in the case of general potentials for which no analytical solutions are known.

The efficiency of Quasi Sturmian functions as basis set has been shown through a simple twobody scattering problem. The capability of the proposed QS basis is related, on one hand, to the range of the driven term (essentially to the range of the functions $\varphi_{n, l}(\lambda, r)$ ) and, on the other hand, to the range of the interaction to be solved. When the latter range is much larger than the former, the QS basis has to have a range larger than the size of the driven term and the number of $\varphi_{n, l}(\lambda, r)$ has to be sufficient to allow the QS to reach far away regions. This means that the necessary number of basis elements will depend mostly on the range of the interaction.

This contribution had not the intention to directly address the three-body problem but to discuss the properties of basis functions that will be used to generate, later on, their solutions. In that sense, it is planned to use the proposed two-body QS basis set to tackle three-body problems, similarly to what has been done with GSFs (see the Introduction). Two different lines will be followed. One of them consists in taking advantage of the QS analytical nature to investigate how the three-body hyperspherical front is actually generated when spherical coordinates are used. A second approach is based on the construction and use of analytical QS functions similar to the GSFs proposed in Ref. 34, i.e., in hyperspherical coordinates and possessing the correct asymptotic behavior. With such a property, only the inner region (where the driven term is located) needs to be to expanded. Moreover, the basis being analytical, closed form integration on the hyper-radial coordinate may be performed, reducing in this way the dimensionality of the problem.

Finally, the finite difference method briefly described in Sec. IV will be further used to generate numerically QS with potentials which are more general than the Coulomb one. Again, the main objective remains applications to scattering problems involving at least three particles.

\section{ACKNOWLEDGMENTS}

One of the authors (G.G.) thanks the support by PGI (24/F049) of the Universidad Nacional del Sur. G.G. acknowledges also the support of ANPCyT (PICT08/0934) (Argentina) and PIP 200901/552 CONICET (Argentina). This work has been developed within the activities planned in the French-Argentinian programme ECOS-Sud A10E01.

${ }^{1}$ T. N. Rescigno, M. Baertschy, W. A. Isaacs, and C. W. McCurdy, Science 286, 2474 (1999).

${ }^{2}$ I. Bray, D. V. Fursa, A. S. Kadyrov, A. T. Stelbovics, A. S. Kheifets, and A. M. Mukhamedzhanovc, Phys. Rep. 520, 135 (2012).

${ }^{3}$ M. Baertschy, T. N. Rescigno, and C. W. McCurdy, Phys. Rev. A 64, 022709 (2001). 
${ }^{4}$ J. Colgan and M. S. Pindzola, Phys. Rev. A 74, 012713 (2006).

${ }^{5}$ A. S. Kadyrov, A. M. Mukhamedzhanov, A. T. Stelbovics, and I. Bray, Phys. Rev. A 70, 062703 (2004).

${ }^{6}$ I. Bray, D. V. Fursa, A. S. Kheifets, and A. T. Stelbovics, J. Phys. B 35, R117 (2002).

${ }^{7}$ V. A. Knyr, V. V. Nasyrov, and Yu. V. Popov, Correlation and Polarization in Photonic, Electronic, and Atomic Collisions, AIP Conference Proceedings Vol. 697, edited by G. F. Hanne et al. (AIP, Melville, 2003), p 76.

${ }^{8}$ A. L. Frapiccini, J. M. Randazzo, G. Gasaneo, and F. D. Colavecchia, J. Phys. B 43, 101001 (2010)

${ }^{9}$ G. Gasaneo, L. U. Ancarani, D. M. Mitnik, J. M. Randazzo, A. L. Frapiccini, and F. D. Colavecchia, Adv. Quantum Chem. 67, 153 (2013).

${ }^{10}$ C. W. McCurdy, M. Baertschy, and T. M. Rescigno, J. Phys. B 37, R137-R187 (2004).

${ }^{11}$ A. Temkin, Phys. Rev. 126, 130 (1962).

${ }^{12}$ R. Poet, J. Phys. B 11, 3081 (1978).

${ }^{13}$ A. T. Stelbovics, Phys. Rev. Lett. 83, 1570 (1999).

${ }^{14}$ C. W. McCurdy, D. A. Horner, and T. M. Rescigno, Phys. Rev. A 65, 042714 (2002).

${ }^{15}$ M. S. Pindzola and F. Robicheaux, Phys. Rev. A 55, 4617 (1997).

${ }^{16}$ C. W. McCurdy, D. A. Horner, T. N. Rescigno, and F. Martín, Phys. Rev. A 69, 032707 (2004).

${ }^{17}$ J. M. Randazzo, F. Buezas, A. L. Frapiccini, F. D. Colavecchia, and G. Gasaneo, Phys. Rev. A 84, 052715 (2011).

${ }^{18}$ D. M. Mitnik, F. D. Colavecchia, G. Gasaneo, and J. M. Randazzo, Comput. Phys. Commun. 182, 1145 (2011)

${ }^{19}$ G. Gasaneo, D. M. Mitnik, J. M. Randazzo, L. U. Ancarani, and F. D. Colavecchia, Phys. Rev. A 87, 042707 (2013).

${ }^{20}$ G. Gasaneo and L. U. Ancarani, Phys. Rev. A 82, 042706 (2010).

${ }^{21}$ L. U. Ancarani and G. Gasaneo, J. At. Mol. Sci. 2, 203 (2011).

${ }^{22}$ L. D. Landau and E. M. Lifshitz, Quantum Mechanics: Non-Relativistic Theory (Pergamon, Oxford, 1965).

${ }^{23}$ I. S. Gradshteyn and I. M. Ryzhik, Table of Integrals, Series, and Products (Academic Press, NY, 1994).

${ }^{24}$ M. Abramowitz and I. A. Stegun, Handbook of Mathematical Functions (Dover, New York, 1972).

${ }^{25}$ L. U. Ancarani and G. Gasaneo, J. Math. Phys. 49, 063508 (2008).

${ }^{26}$ L. Hostler, J. Math. Phys. 5, 591 (1964).

${ }^{27}$ E. J. Heller, Phys. Rev. A 12, 1222 (1975).

${ }^{28}$ F. Demir, Z. T. Hlousek, and Z. Papp, Phys. Rev. A 74, 014701 (2006).

${ }^{29}$ H. A. Yamani and L. Fishman, J. Math. Phys. 16, 410 (1975).

${ }^{30}$ J. T. Broad and W. Reinhardt, J. Phys. B 9, 1491 (1976).

${ }^{31}$ J. T. Broad, Phys. Rev. A 31, 1494 (1985).

${ }^{32}$ See www.netlib.org/lapack for Linear Algebra Package.

${ }^{33}$ M. J. Ambrosio, J. A. Del Punta, K. V. Rodriguez, G. Gasaneo, and L. U. Ancarani, J. Phys. A 45, 015201 (2012).

${ }^{34}$ L. U. Ancarani, G. Gasaneo, and D. M. Mitnik, Eur. Phys. J. D 66, 270 (2012). 